\title{
Numerical simulation of tidal flow in Danang Bay Based on non-hydrostatic shallow water equations
}

\author{
Thi Thai Le $\mathbb{E}^{*}$, Dang Hieu Phung ${ }^{2}$ and Van Cuc Tran ${ }^{3}$
}

\begin{abstract}
This paper presents a numerical simulation of the tidal flow in Danang Bay (Vietnam) based on the non-hydrostatic shallow water equations. First, to test the simulation capability of the non-hydrostatic model, we have made a test simulation comparing it with the experiment by Beji and Battjes 1993, Coastal Engineering 23, 1-16. Simulation results for this case are compared with both the experimental data and calculations obtained from the traditional hydrostatic model. It is shown that the non-hydrostatic model is better than the hydrostatic model when the seabed topography variation is complex. The usefulness of the non-hydrostatic model is father shown by successfully simulating the tidal flow of Danang Bay.
\end{abstract}

Keywords: Non-hydrostatic shallow water equations, Tidal flow, Numerical method

\section{Introduction}

The effect of non-hydrostatic pressure on the shallowwater model has been a topic of interest for the shallowwater to simulate long waves such as tides, storm surges, and tsunamis. The finite difference method is widely used in the solution schemes of the depth-integrated governing equations. Researchers have made an effort for improving numerical schemes and boundary treatments to model long-wave propagation, transformation, and run-up.

Stelling and Duinmeijer [14] provided a numerical technique that in essence is based upon the classical staggered grids and implicit numerical integration schemes, but that can be applied to problems that include rapidly varied flows as well. They imposed energy conservation to strong flow contractions and momentum conservation to mild flow contractions and expansions. Their approach approximates flow discontinuities as bores or hydraulic jumps as in a finite volume model, using a Riemann solver [4, 5, 22]. Horrillo et al. [6] showed some of the implications of dispersion effects in tsunami propagation and run-up through the processes as follow: first, a brief

*Correspondence: lethithai1208@gmail.com

1 Hanoi University of Natural Resources and Environment, Hanoi, Vietnam

Full list of author information is available at the end of the article description of the model formulation and their numerical schemes is presented. Therefore, several numerical experiments are described with initial conditions for free surface deformations. Then, model results are compared against each other. Finally, observations and model results are analyzed to draw conclusion on the spatial and temporal distributions of the free surface.

Stelling and Zijlema [15] employed the Keller-box method that takes into account the effect of nonhydrostatic pressure of free-surface flows with a very small number of vertical grid points to approximate the vertical gradient of the pressure arising in the Reynolds-averaged NavierStokes equations. In both the depth-integrated and multi-layer formulations, they decompose the pressure into hydrostatic and non-hydrostatic components following Casulli [2] and apply the Keller-box scheme [7] to the vertical gradient approximation of the non-hydrostatic pressure. Then they proposed a semi-implicit finite difference scheme, which accounts for dispersion through a non-hydrostatic pressure term. Walters [18] adapted this non-hydrostatic approach to a finite element method. His numerical results showed that both depth-integrated models estimate the dispersive waves slightly better than the classical Boussinesq equations by Peregrine [13]. Zijlema and Stelling [23] recently extended their multi-

\section{Springer}

(c) 2016 Le et al. Open Access This article is distributed under the terms of the Creative Commons Attribution 4.0 International License (http://creativecommons.org/licenses/by/4.0/), which permits unrestricted use, distribution, and reproduction in any medium, provided you give appropriate credit to the original author(s) and the source, provide a link to the Creative Commons license, and indicate if changes were made. 
layer model without using of empirical relations to energy dissipation by using a compact finite difference scheme. Their accurate computation of frequency dispersion saves vertical resolution and hence is capable of predicting the onset of wave breaking. A novel wet-dry algorithm is applied for a proper handling of moving shoreline. Mass and momentum are strictly conserved at discrete level while the method allow for energy dissipation only in the case of wave breaking and provide comparable results with those of extended Boussinesq models $[3,10,12]$. Mader [11] proposed a unique upwind scheme that extrapolates the surface elevation instead of the flow depth to determine explicitly the flux in the continuity equation of a nonlinear shallow-water model. Kowalik et al. [8] implemented this upwind flux approximation and showed remarkable stability in simulating global tsunami propagation and run-up. It exhibits high-order accuracy for the spatial derivatives. The boundary condition at the shore line is controlled by the total depth and can be set either to runup or to the zero normal velocity. Yamazaki et al. [19] have proposed a depth-integrated non-hydrostatic model which is capable of handling flow discontinuities associated with wave breaking and hydraulic jumps. Their model builds on an explicit scheme of the nonlinear shallow-water equations and makes possible a direct implementation of the upwind flux approximation of Kowalik et al. [8] in order to improve model stability for discontinuous flow. The improved model includes an empirical coefficient $\alpha(\alpha \geq 0)$. In their treatment, the empirical coefficient $\alpha$ related to the depth-integrated non-hydrostatic pressure is approximated by 0.5 .

Wei and Jia [21] provided a two-dimensional finite element method based on the FEM platform of CCHE2D for simulating dynamic propagation of weakly dispersive waves. A physically bounded upwind scheme for discretizing the advection term is developed, and the quasi second-order differential operators of this scheme result in no oscillation and little numerical diffusion.

The purpose of this paper is to present a formulation of a new general non-hydrostatic shallow-water equations with disposable parameter related to integration of the pressure in the depth. Section 2 provides the equations augmented with parameter $\alpha(\alpha \geq 0)$ by applying the Leibniz rule for the non-hydrostatic pressure component together with the boundary conditions. The parameter $\alpha$ is determined by the vertical average of $f(z)$ with $\alpha=$ 0 corresponding to the hydrostatic approximation. The numerical model is also presented in Section 2 through three main steps as follows: The first is an explicit method for hydrostatic component of the pressure, and the second is a formulation of the Poisson equation for implicitly solving the non-hydrostatic pressure. Then the third is their combination. Section 3 presents the results of the application of our non-hydrostatic model to simulate tidal flow. Section 3 makes a comparison between the nonhydrostatic model and the traditional hydrostatic model for laboratory experiment of sinusoidal wave propagation over a submerged barrier in a wave channel. In particular, our model is applied to simulate actual tidal flows in Danang Bay (Vietnam), successfully obtaining good agreement with measured data. Section 4 presents discussion in a wider viewpoint and proposes further development with other conditions to simulation in various other problems, such as: tides, storm surges and tsunamis, etc.

\section{Derivation of the model for shallow water flow with non-hydrostatic pressure}

\subsection{The governing equations}

We assume an incompressible fluid in a uniform environment. Then, we can write the Navier-Stokes equations as

$$
\frac{\partial u}{\partial t}+u \frac{\partial u}{\partial x}+v \frac{\partial u}{\partial y}+\mathrm{w} \frac{\partial u}{\partial z}=-\frac{1}{\rho} \frac{\partial P}{\partial x}+v\left(\frac{\partial^{2} u}{\partial x^{2}}+\frac{\partial^{2} u}{\partial y^{2}}+\frac{\partial^{2} u}{\partial z^{2}}\right)
$$

$$
\frac{\partial v}{\partial t}+u \frac{\partial v}{\partial x}+v \frac{\partial v}{\partial y}+\mathrm{w} \frac{\partial v}{\partial z}=-\frac{1}{\rho} \frac{\partial P}{\partial y}+v\left(\frac{\partial^{2} v}{\partial x^{2}}+\frac{\partial^{2} v}{\partial y^{2}}+\frac{\partial^{2} v}{\partial z^{2}}\right),
$$

$$
\begin{aligned}
\frac{\partial \mathrm{w}}{\partial t} & +u \frac{\partial \mathrm{w}}{\partial x}+v \frac{\partial \mathrm{w}}{\partial y}+\mathrm{w} \frac{\partial \mathrm{w}}{\partial z}=-\frac{1}{\rho} \frac{\partial P}{\partial z} \\
& +v\left(\frac{\partial^{2} \mathrm{w}}{\partial x^{2}}+\frac{\partial^{2} \mathrm{w}}{\partial y^{2}}+\frac{\partial^{2} \mathrm{w}}{\partial z^{2}}\right)-g .
\end{aligned}
$$

The continuity equation is given by

$$
\frac{\partial u}{\partial x}+\frac{\partial v}{\partial y}+\frac{\partial \mathrm{w}}{\partial z}=0
$$

and the surface equation is

$$
\zeta=\zeta(x, y, t)
$$

where $(u, v, w)$ are the velocity components in the horizontal ( $x$ and $y-)$ directions and in the vertical ( $z-$ ) direction, respectively. $t$ is time, $\rho$ is the density of water, $v$ is the kinetic viscosity coefficient; $P$ is the pressure, and $g$ is the gravitational acceleration.

The pressure at any point is divided into two components: the hydrostatic pressure and the non-hydrostatic pressure, as follows [20]:

$$
P=\rho g(\zeta-z)+\hat{q}(x, y, z, t),
$$

where, $\hat{q}(x, y, z, t)$ is defined as the non-hydrostatic pressure.

We integrate the component of the momentum equation in the vertical coordinate from the bottom 
$(z=-h)$ to the surface $(z=\zeta)$. We introduce the definition of the vertical average of any quantity $f$ as

$$
F=\frac{1}{D} \int_{-h}^{\zeta} f d z
$$

where $\zeta$ is the surface elevation height, $h$ is the still-water depth and $D=\zeta+h$ is the total water depth from the bottom to the surface. We obtain

$$
\begin{aligned}
& \int_{-h}^{\zeta}\left(\frac{\partial u}{\partial t}+u \frac{\partial u}{\partial x}+v \frac{\partial u}{\partial y}+\mathrm{w} \frac{\partial u}{\partial z}\right) d z \\
& =-\frac{1}{\rho} \int_{-h}^{\zeta} \frac{\partial P}{\partial x} d z+v \int_{-h}^{\zeta}\left(\frac{\partial^{2} u}{\partial x^{2}}+\frac{\partial^{2} u}{\partial y^{2}}+\frac{\partial^{2} u}{\partial z^{2}}\right) d z
\end{aligned}
$$

The term $\int_{-h}^{\zeta} \mathrm{w} \frac{\partial u}{\partial z} d z$ is very small and can be neglected. For wave motion, we assume that the fluid velocity is small, so the viscosity term $v \int_{-h}^{\zeta}\left(\frac{\partial^{2} u}{\partial x^{2}}+\frac{\partial^{2} u}{\partial y^{2}}\right) d z$ is small relative to the advection term and can therefore be neglected too.

We consider the first term of the right-hand side

$$
\begin{aligned}
-\frac{1}{\rho} \int_{-h}^{\zeta} \frac{\partial P}{\partial x} d z & =-\frac{1}{\rho} \int_{-h}^{\zeta} \frac{\partial}{\partial x}[\rho g(\zeta-z)+\hat{q}] d z \\
& =-g D \frac{\partial \zeta}{\partial x}-\frac{1}{\rho} \int_{-h}^{\zeta} \frac{\partial \hat{q}}{\partial x} d z .
\end{aligned}
$$

In order to generalize Yamazaki et al. (2008), we represent the non-hydrostatic component of the pressure by introducing some function as

$$
\hat{q}(x, y, z, t)=q(x, y,-h, t) f(z),
$$

where $f_{-h}=f(-h)=1$. Then, by applying the Leibniz rule, we have

$$
\int_{-h}^{\zeta} \frac{\partial \hat{q}}{\partial x} d z=\frac{\partial}{\partial x} \int_{-h}^{\zeta} \hat{q} d z-\hat{q}_{\zeta} \frac{\partial \zeta}{\partial x}+\hat{q}_{-h} \frac{\partial(-h)}{\partial x} .
$$

Then, we obtain

$$
\int_{-h}^{\zeta} \frac{\partial \hat{q}}{\partial x} d z=\frac{\partial}{\partial x} q \int_{-h}^{\zeta} f d z+0+q_{-h} f_{-h} \frac{\partial(-h)}{\partial x},
$$

with $\hat{q}_{\zeta}=0$ since the total pressure vanishes at the free surface. We introduce parameter $\alpha$ by $\alpha=\frac{1}{D} \int_{-h}^{\zeta} f d z$. We see that $\alpha$ is an empirical coefficient related to the depthintegrated non-hydrostatic pressure and can be expressed as the vertical average of $f(z)$. Equation (12) then become

$$
\int_{-h}^{\zeta} \frac{\partial \hat{q}}{\partial x} d z=D \frac{\partial}{\partial x}(\alpha q)+q\left(\alpha \frac{\partial \zeta}{\partial x}+(1-\alpha) \frac{\partial(-h)}{\partial x}\right) .
$$

If $f(z)$ is taken to be a linear function, the value of $\alpha$ is $1 / 2$. The non-hydrostatic pressure at the bottom has been written simply as $q$ now.

$$
\begin{aligned}
-\frac{1}{\rho} \int_{-h}^{\zeta} \frac{\partial P}{\partial x} d z= & -\frac{1}{\rho} \int_{-h}^{\zeta} \frac{\partial}{\partial x}[\rho g(\zeta-z)+\hat{q}] d z \\
= & -g D \frac{\partial \zeta}{\partial x}-\frac{1}{\rho} D \alpha \frac{\partial q}{\partial x}-\alpha \frac{q}{\rho} \frac{\partial \zeta}{\partial x} \\
& -(1-\alpha) \frac{q}{\rho} \frac{\partial(-h)}{\partial x}
\end{aligned}
$$

Finally, after integration, the first momentum equation in the $\mathrm{x}$ direction results in

$$
\begin{aligned}
\frac{\partial U}{\partial t}+U \frac{\partial U}{\partial x}+V \frac{\partial U}{\partial y}= & -g \frac{\partial \zeta}{\partial x}-\frac{1}{\rho} \alpha \frac{\partial q}{\partial x}-\alpha \frac{q}{\rho D} \frac{\partial \zeta}{\partial x} \\
& -(1-\alpha) \frac{q}{\rho D} \frac{\partial(-h)}{\partial x}-\frac{g}{C_{z}^{2}} \frac{U \sqrt{U^{2}+V^{2}}}{\rho D},
\end{aligned}
$$

where $U$ and $V$ are the vertical averages of $u$ and $v$ and the last term originates from the friction force at the bottom. Here, $C_{z}$ is a constant called the Chezy coefficient.

Similarly, the second momentum Eq. (2) in the $y$ direction gives

$$
\begin{aligned}
\frac{\partial V}{\partial t}+U \frac{\partial V}{\partial x}+V \frac{\partial V}{\partial y}= & -g \frac{\partial \zeta}{\partial y}-\frac{1}{\rho} \alpha \frac{\partial q}{\partial y}-\alpha \frac{q}{\rho D} \frac{\partial \zeta}{\partial y} \\
& -(1-\alpha) \frac{q}{\rho D} \frac{\partial(-h)}{\partial y}-\frac{g}{C_{z}^{2}} \frac{V \sqrt{U^{2}+V^{2}}}{\rho D}
\end{aligned}
$$

There are generalization of Yamazaki et al. (2008) to introduce a disposal parameter $\alpha$. 
Integrating the third momentum Eq. (3) in the $\mathrm{z}$ direction (3) we obtain

$$
\begin{aligned}
\int_{-h}^{\zeta} \frac{\partial \mathrm{w}}{\partial t} d z & +\int_{-h}^{\zeta}\left(u \frac{\partial \mathrm{w}}{\partial x}+v \frac{\partial \mathrm{w}}{\partial y}+\mathrm{w} \frac{\partial \mathrm{w}}{\partial z}\right) d z=-\frac{1}{\rho} \int_{-h}^{\zeta} \frac{\partial P}{\partial z} d z \\
& +v \int_{-h}^{\zeta}\left(\frac{\partial^{2} \mathrm{w}}{\partial x^{2}}+\frac{\partial^{2} \mathrm{w}}{\partial y^{2}}+\frac{\partial^{2} \mathrm{w}}{\partial z^{2}}\right) d z-g \int_{-h}^{\zeta} d z
\end{aligned}
$$

For propagation of long wave of large scale, the viscous term is negligible and so the terms $v \int_{-h}^{\zeta}\left(\frac{\partial^{2} \mathrm{w}}{\partial x^{2}}+\frac{\partial^{2} \mathrm{w}}{\partial y^{2}}+\frac{\partial^{2} \mathrm{w}}{\partial z^{2}}\right) d z$ is neglected.

At the right-hand side of Eq. (17), after neglecting the above terms, we have

$$
\begin{aligned}
-\frac{1}{\rho} \int_{-h}^{\zeta} \frac{\partial P}{\partial z} d z-\int_{-h}^{\zeta} g d z & =-\frac{1}{\rho}\left[\left.P\right|_{z=\zeta}-\left.P\right|_{z=-h}\right]-g D \\
& =-\frac{1}{\rho}\left[\left.\hat{q}\right|_{z=\zeta}-\left.\hat{q}\right|_{z=-h}\right]=\frac{\hat{q}_{z=-h}}{\rho}=\frac{q}{\rho} .
\end{aligned}
$$

Where, $q$ is again the non-hydrostatic pressure at the bottom as in the momentum equations in the horizontal directions $x$ and $y$ has a link with $\hat{q}$ through (10). We recall that $\left.\hat{q}\right|_{z=\zeta}=0$ since the total pressure vanishes at the free surface. The third momentum equation, in the $z$ direction, after integrating results in $z$, become

$$
\frac{\partial \mathrm{W}}{\partial t}=\frac{q}{\rho D}
$$

Integrating the continuity equation, we obtain

$$
D \frac{\partial U}{\partial x}+D \frac{\partial V}{\partial y}+\left.\mathrm{w}\right|_{z=\zeta}-\left.\mathrm{w}\right|_{z=-h}=0 .
$$

Using the boundary conditions

$$
\left.\mathrm{w}\right|_{z=-h}=\frac{d(-h)}{d t}=-u \frac{\partial h}{\partial x}-v \frac{\partial h}{\partial y},
$$

at the bottom

$$
\left.\mathrm{w}\right|_{z=\zeta}=\frac{d(\zeta)}{d t}=\frac{\partial \zeta}{\partial t}+u \frac{\partial \zeta}{\partial x}+v \frac{\partial \zeta}{\partial y},
$$

at the surface and substituting them into Eq. (20), we obtain

$$
\frac{\partial \zeta}{\partial t}+\frac{\partial(U D)}{\partial x}+\frac{\partial(V D)}{\partial y}=0
$$

We have eventually the governing equations with the effect of non-hydrostatic pressure for an incompressible fluid in uniform environments as the system of Eqs. (15),
(16), (19) and (23). Because the distribution of the vertical velocity is unknown, it is approximated by [17]:

$$
\mathrm{W}=\frac{\mathrm{W}_{\zeta}+\mathrm{W}_{-h}}{2}=\frac{\mathrm{W}_{s}+\mathrm{W}_{b}}{2} .
$$

\subsection{Boundary conditions}

Here, we use five the boundary conditions as follow:

$\boldsymbol{i}$. Free surface: The wind stress and the surface tension are not considered. Only the atmospheric pressure is imposed on the free surface level.

ii. Bottom: The vertical velocity at the bottom is calculated from the kinematic boundary condition (21).

iii. We consider source waves in the form

$$
\zeta=A \cos \frac{2 \pi t}{T}
$$

where $\mathrm{A}$ is the wave amplitude, $\mathrm{T}$ is the wave period.

iv. Neuman condition is imposed at the end of a river where the flow enters the sea.

$$
\frac{\partial \vec{u}}{\partial \vec{n}}=0
$$

v. The impermeable boundary enforces

$$
u_{n}=0
$$

along the coast or at the wall.

\subsection{Numerical model}

The input of the problem are the initial conditions $U_{0}, V_{0}, \mathrm{~W}_{0}, \zeta_{0}, q_{0}$, physical constants and relative conditions. The outputs are the horizontal velocity components $U$ and $V$, the vertical velocity $W$, the surface elevation $\zeta$, and the non-hydrostatic pressure component $q$, where the water depth $h$ is defined. Figure 1 shows the spatial grid for computation.

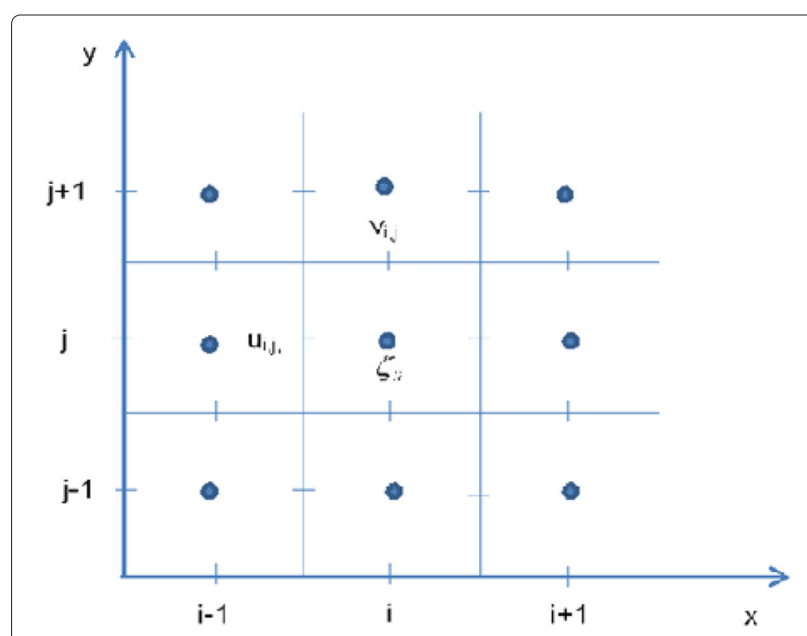

Fig. 1 The difference grid map 
In the first step, we use an explicit method for the hydrostatic components as follows

$$
\begin{aligned}
& \frac{\partial U}{\partial t}+U \frac{\partial U}{\partial x}+V \frac{\partial U}{\partial y}=-g \frac{\partial \zeta}{\partial x}-\frac{g}{C_{z}^{2}} \frac{U \sqrt{U^{2}+V^{2}}}{\rho D}, \\
& \frac{\partial V}{\partial t}+U \frac{\partial V}{\partial x}+V \frac{\partial V}{\partial y}=-g \frac{\partial \zeta}{\partial y}-\frac{g}{C_{z}^{2}} \frac{V \sqrt{U^{2}+V^{2}}}{\rho D} .
\end{aligned}
$$

For the horizontal velocity components we have

$$
\begin{aligned}
U_{i, j}^{m+1}= & U_{i, j}^{m}-g \frac{\Delta t}{\Delta x}\left(\zeta_{i, j}^{m}-\zeta_{i-1, j}^{m}\right)-\frac{\Delta t}{\Delta x} U_{p}^{m}\left(U_{i, j}^{m}-U_{i-1, j}^{m}\right) \\
& -\frac{\Delta t}{\Delta x} U_{n}^{m}\left(U_{i+1, j}^{m}-U_{i, j}^{m}\right)-\frac{\Delta t}{\Delta y} \bar{V}_{y_{p}}^{m}\left(U_{i, j}^{m}-U_{i, j-1}^{m}\right) \\
& -\frac{\Delta t}{\Delta y} \bar{V}_{x_{n}}^{m}\left(U_{i, j+1}^{m}-U_{i, j}^{m}\right)-\frac{g}{C_{z}^{2}} \frac{\Delta t U_{i, j}^{m} \sqrt{\left(U_{i, j}^{m}\right)^{2}+\left(\bar{V}_{x_{i, j}}^{m}\right)^{2}}}{D_{i-1, j}^{m}+D_{i, j}^{m}},
\end{aligned}
$$

$$
\begin{aligned}
V_{i, j}^{m+1}= & V_{i, j}^{m}-g \frac{\Delta t}{\Delta y}\left(\zeta_{i, j+1}^{m}-\zeta_{i, j}^{m}\right)-\frac{\Delta t}{\Delta x} \bar{U}_{y_{p}}^{m}\left(V_{i, j}^{m}-V_{i-1, j}^{m}\right) \\
& -\frac{\Delta t}{\Delta x} \bar{U}_{y_{n}}^{m}\left(V_{i+1, j}^{m}-V_{i, j}^{m}\right)-\frac{\Delta t}{\Delta y} V_{p}^{m}\left(V_{i, j}^{m}-V_{i, j-1}^{m}\right) \\
& -\frac{\Delta t}{\Delta y} V_{n}^{m}\left(V_{i, j+1}^{m}-V_{i, j}^{m}\right)-\frac{g}{C_{z}^{2}} \frac{\Delta t V_{i, j}^{m} \sqrt{\left(\bar{U}_{y_{i, j}}^{m}\right)^{2}+\left(V_{i, j}^{m}\right)^{2}}}{D_{i, j}^{m}+D_{i, j+1}^{m}},
\end{aligned}
$$

where superscript $m$ denotes the time, subsript $p$ and $n$ describe a positive flow and a negative flow respectively, and $\bar{V}_{x_{p}}^{m}, \bar{V}_{x_{n}}^{m}, \bar{U}_{y_{p}}^{m}$, and $\bar{U}_{y_{n}}^{m}$ which were defined in the model of Yamazaki et al. [19] are advective speeds in the respective $y$ - and $x$-momentum equations. The value of the depth-averaged velocity components $U$ and $V$ used in the momentum equations in the $\mathrm{x}$ and $\mathrm{y}$ directions are respectively defined by [19]

$$
\begin{aligned}
& \bar{U}_{y_{i, j}}^{m}=\frac{1}{4}\left(U_{i, j}+U_{i+1, j}+U_{i+1, j+1}+U_{i, j+1}\right), \\
& \bar{V}_{x_{i, j}}^{m}=\frac{1}{4}\left(V_{i, j}+V_{i-1, j}+V_{i-1, j-1}+V_{i, j-1}\right) .
\end{aligned}
$$

In the second step, we will present a formulation where the Poisson equation is implicitly solved for the nonhydrostatic pressure. The final velocity is updated with the non-hydrostatic pressure.

Discretization of the vertical momentum Eq. (19) given the vertical velocity at the free surface as

$$
\mathrm{W}_{s_{i, j}}^{m+1}=\mathrm{W}_{s_{i, j}}^{m}-\left(\mathrm{W}_{b_{i, j}}^{m+1}-\mathrm{W}_{b_{i, j}}^{m}\right)+\frac{2 \Delta t}{\rho D_{i, j}^{m}} q_{i, j}^{m+1},
$$

where $\mathrm{W}_{s_{i, j}}^{m}$ and $\mathrm{W}_{b_{i, j}}^{m}$ are the vertical velocities at time $\mathrm{m}$, at the surface and the bottom respectively. The horizontal velocities influenced by the non-hydrostatic pressure are expressed as

$$
\begin{aligned}
U_{i, j}^{m+1}= & \tilde{U}_{i, j}^{m+1}-\frac{\Delta t}{\rho \Delta x} \frac{\left(q_{i, j}^{m+1}+q_{i-1, j}^{m+1}\right)}{\left(D_{i, j}^{m}+D_{i-1, j}^{m}\right)} \\
& \times\left[\alpha\left(\zeta_{i, j}^{m}-\zeta_{i-1, j}^{m}\right)+(1-\alpha)\left(h_{i, j}-h_{i-1, j}\right)\right] \\
& -\frac{\Delta t}{\rho \Delta x} \alpha\left(q_{i, j}^{m+1}-q_{i-1, j}^{m+1}\right),
\end{aligned}
$$

$$
\begin{aligned}
V_{i, j}^{m+1}= & \tilde{V}_{i, j}^{m+1}-\frac{\Delta t}{\rho \Delta y} \frac{\left(q_{i, j+1}^{m+1}+q_{i, j}^{m+1}\right)}{\left(D_{i, j}^{m}+D_{i, j+1}^{m}\right)} \\
& \times\left[\alpha\left(\zeta_{i, j+1}^{m}-\zeta_{i, j}^{m}\right)+(1-\alpha)\left(h_{i, j+1}-h_{i, j}\right)\right] \\
& -\frac{\Delta t}{\rho \Delta y} \alpha\left(q_{i, j+1}^{m+1}-q_{i, j}^{m+1}\right) .
\end{aligned}
$$

The continuity equation is directly applied for the depth-averaged water column,

$$
\frac{U_{i+1, j}^{m+1}-U_{i, j}^{m+1}}{\Delta x}+\frac{V_{i, j}^{m+1}-V_{i, j-1}^{m+1}}{\Delta y}+\frac{\mathrm{W}_{s_{i, j}}^{m+1}-\mathrm{W}_{b_{i, j}}^{m+1}}{D_{i, j}^{m}}=0,
$$

The vertical velocity at the bottom is calculated from the boundary condition in Eq. (21) as

$$
\begin{aligned}
\mathrm{W}_{b_{i, j}}^{m+1}= & -\bar{U}_{z_{p}}^{m} \frac{h_{i, j}-h_{i-1, j}}{\Delta x}-\bar{U}_{z_{n}}^{m} \frac{h_{i+1, j}-h_{i, j}}{\Delta x}-\bar{V}_{z_{p}}^{m} \frac{h_{i, j}-h_{i, j-1}}{\Delta y} \\
& -\bar{V}_{z_{n}}^{m} \frac{h_{i, j+1}-h_{i, j}}{\Delta y} .
\end{aligned}
$$

Finally, we obtain the Poisson equation to find the nonhydrostatic pressure as

$$
\begin{aligned}
A P_{i, j} q_{i-1, j}^{m+1} & +A W_{i, j} q_{i+1, j}^{m+1}+A E_{i, j} q_{i, j-1}^{m+1}+A N_{i, j} q_{i, j-1}^{m+1} \\
& +A S_{i, j} q_{i, j}^{m+1}=S_{i, j},
\end{aligned}
$$

where

$$
\begin{aligned}
& \frac{\Delta t}{\rho \Delta x^{2}}\left(-\alpha+A_{i, j}\right)=A P_{i, j}, \quad \frac{\Delta t}{\rho \Delta x^{2}}\left(-\alpha-A_{i+1, j}\right)=A W_{i, j}, \\
& \frac{\Delta t}{\rho \Delta y^{2}}\left(-\alpha+B_{i, j-1}\right)=A E_{i, j}, \quad \frac{\Delta t}{\rho \Delta y^{2}}\left(-\alpha-B_{i, j}\right)=A N_{i, j} \text {, } \\
& \frac{\Delta t}{\rho \Delta x^{2}}\left(\alpha+A_{i, j}-A_{i+1, j}\right)+\frac{\Delta t}{\rho \Delta y^{2}}\left(1+B_{i, j-1}-B_{i, j}\right) \\
& +\frac{2 \Delta t}{\rho\left(D_{i, j}^{m}\right)^{2}}=A S_{i, j},
\end{aligned}
$$




$$
\begin{gathered}
-\frac{U_{i+1, j}^{m+1}-U_{i, j}^{m+1}}{\Delta x}-\frac{V_{i, j}^{m+1}-V_{i, j-1}^{m+1}}{\Delta y} \\
-\frac{\mathrm{W}_{s_{i, j}}^{m}+\mathrm{W}_{b_{i, j}}^{m}-2 \mathrm{~W}_{b_{i, j}}^{m+1}}{D_{i, j}^{m}}=Q_{i, j}, \\
\frac{\left[\alpha\left(\zeta_{i, j}^{m}-\zeta_{i-1, j}^{m}\right)+(1-\alpha)\left(h_{i, j}-h_{i-1, j}\right)\right]}{\left(D_{i, j}^{m}+D_{i-1, j}^{m}\right)}=A_{i, j}, \\
\frac{\left[\alpha\left(\zeta_{i, j+1}^{m}-\zeta_{i, j}^{m}\right)+(1-\alpha)\left(h_{i, j+1}-h_{i, j}\right)\right]}{\left(D_{i, j}^{m}+D_{i, j+1}^{m}\right)}=B_{i, j} .
\end{gathered}
$$

In the third step, the surface elevation is calculated from the mass conservation Eq. (23) as [9]

$\zeta_{i, j}^{m+1}=\zeta_{i, j}^{m}-\Delta t \frac{\left(F L X_{i+1, j}-F L X_{i, j}\right)}{\Delta x}-\Delta t \frac{\left(F L Y_{i, j}-F L Y_{i, j-1}\right)}{\Delta y}$

where

$$
\left\{\begin{array}{l}
F L X_{i, j}=U_{p}^{m+1} \zeta_{i-1, j}^{m}+U_{n}^{m+1} \zeta_{i, j}^{m}+U_{i, j}^{m+1} \frac{\left(h_{i-1, j}+h_{i, j}\right)}{2} \\
F L Y_{i, j}=V_{p}^{m+1} \zeta_{i, j}^{m}+V_{n}^{m+1} \zeta_{i, j+1}^{m}+V_{i, j}^{m+1} \frac{\left(h_{j, k}+h_{i, j+1}\right)}{2}
\end{array}\right.
$$

\section{Results of the application of the non-hydrostatic model to simulate flow}

3.1 Wave propagation over a submerged barrier in a wave channel.

Figure 2 shows our numerical setup mirroring the experiment of Beji and Battjes [1] to simulate wave propagation over a submerged barrier in a wave flume $37.7 \mathrm{~m}$ long, $0.8 \mathrm{~m}$ wide and $0.75 \mathrm{~m}$ high; the still water height $H$ is $0.4 \mathrm{~m}$, a $0.3 \mathrm{~m}$ high trapezoidal barrier with an offshore slope of 1:20 and a shoreward slope of 1:10 is set between
6.0 and $17.0 \mathrm{~m}$ in the flume and a $1: 25$ plane beach with coarse material. At the left side is an open-flow area modeled by imposing a radiation boundary condition [17]. The incident sinusoidal waves of wave number $k$ have an amplitude of $1.0 \mathrm{~m}$ and a wave period of $2.02 \mathrm{~s}$, corresponding to the water depth parameter $k H=0.67$ and are generated at the left side, based on the linear wave theory. We use mesh sizes $\Delta x=\Delta y=1.25 \mathrm{~cm}$, time interval $\Delta t=0.01 \mathrm{~s}$ and the Courant constant $C r=0.5$. Here, the Courant constant reflects the portion of a cell that a solute will traverse by advection in one time step and the stability of the numerical model is affected by the value of Courant parameter. When advection dominates dispersion, designing a model with a small Courant number will decrease oscillation, improve accuracy and decrease numerical dispersion. The value of Courant parameter changes with the method used to solve the discretised equation, especially depending on whether the finite-difference method for time derivative or the time advancement is explicit or implicit.

Figure 3 shows the calculated results of water waves using the hydrostatic (dashed liner) and non-hydrostatic models (solid liner) for eight experimental datasets. We clearly observe that results of the non-hydrostatic model are almost identical to the experimental data and are far better than the traditional hydrostatic model. In particular, the results calculated under the non-hydrostatic model show that this model can simulate the water fluctuation well while the traditional hydrostatic model could not reproduce the secondary waves at datasets $G_{3}$ to $G_{8}$ marked with the numbere in the above in Fig. 2. The secondary waves are generated due to the effects of the submerged barrier in the wave channel.

\subsection{Numerical simulation of tidal flow in Da Nang Bay, Vietnam}

Now we applied our model to simulate the tidal flow in Danang Bay (Vietnam) including three measuring points of water level fluctuation: Hon Chao (longitude

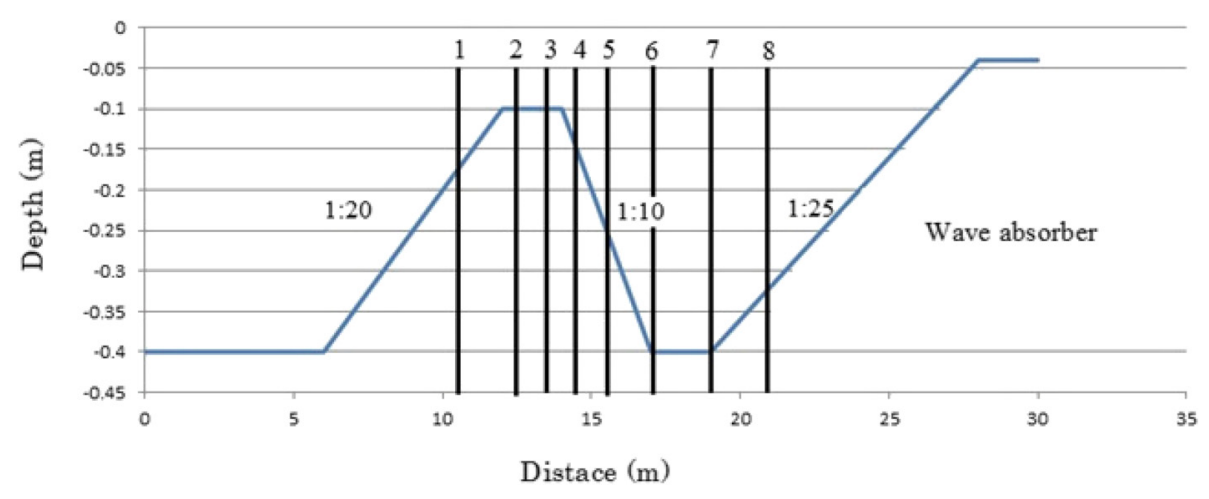

Fig. 2 Numerical model setup of sinusoidal waves propagation over a submerged bar in flume 

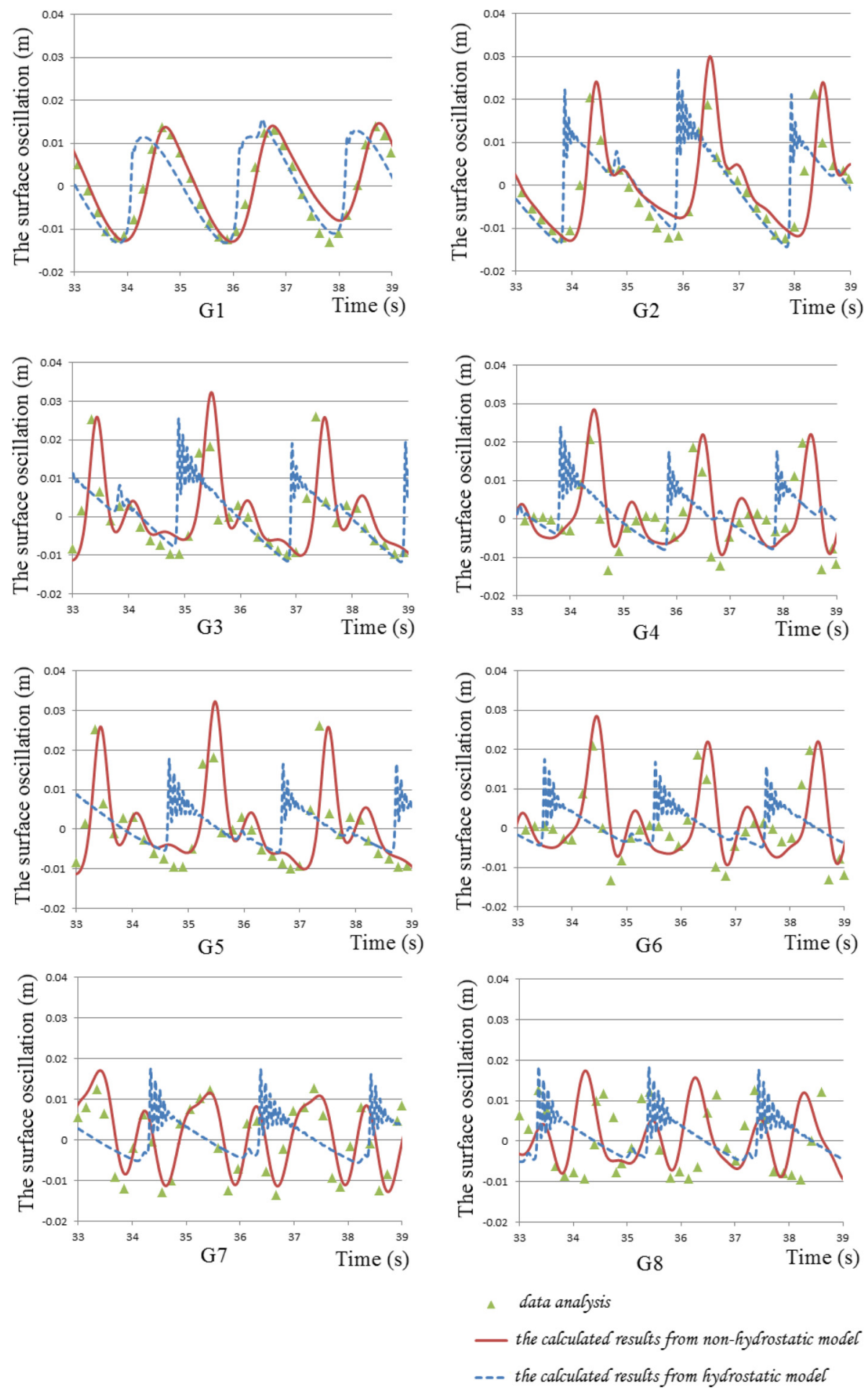

Fig. 3 Comparison of water level fluctuation between the non-hydrostatic and hydrostatic models with experimental data

$108.219^{\circ} \mathrm{E}$, latitude $16.226^{\circ} \mathrm{N}$ ), Cua Vinh (longitude $108.217^{\circ} \mathrm{E}$, latitude $16.177^{\circ} \mathrm{N}$ and Giua Vinh (longitude $108.18^{\circ} \mathrm{E}$, latitude $16.138^{\circ} \mathrm{N}$ ) as in Fig. 4. The coastal length of Danang Bay is $92 \mathrm{~km}$ and is discretized with
$\Delta x=\Delta y=150 m, \Delta t=1 h$, with the maximum Courant number $C r=0.5$, and a friction coefficient $C_{f}=0.002$. The total calculation time is one month (from May 1, 2014 to May 31, 2014). And the tidal source term used is 


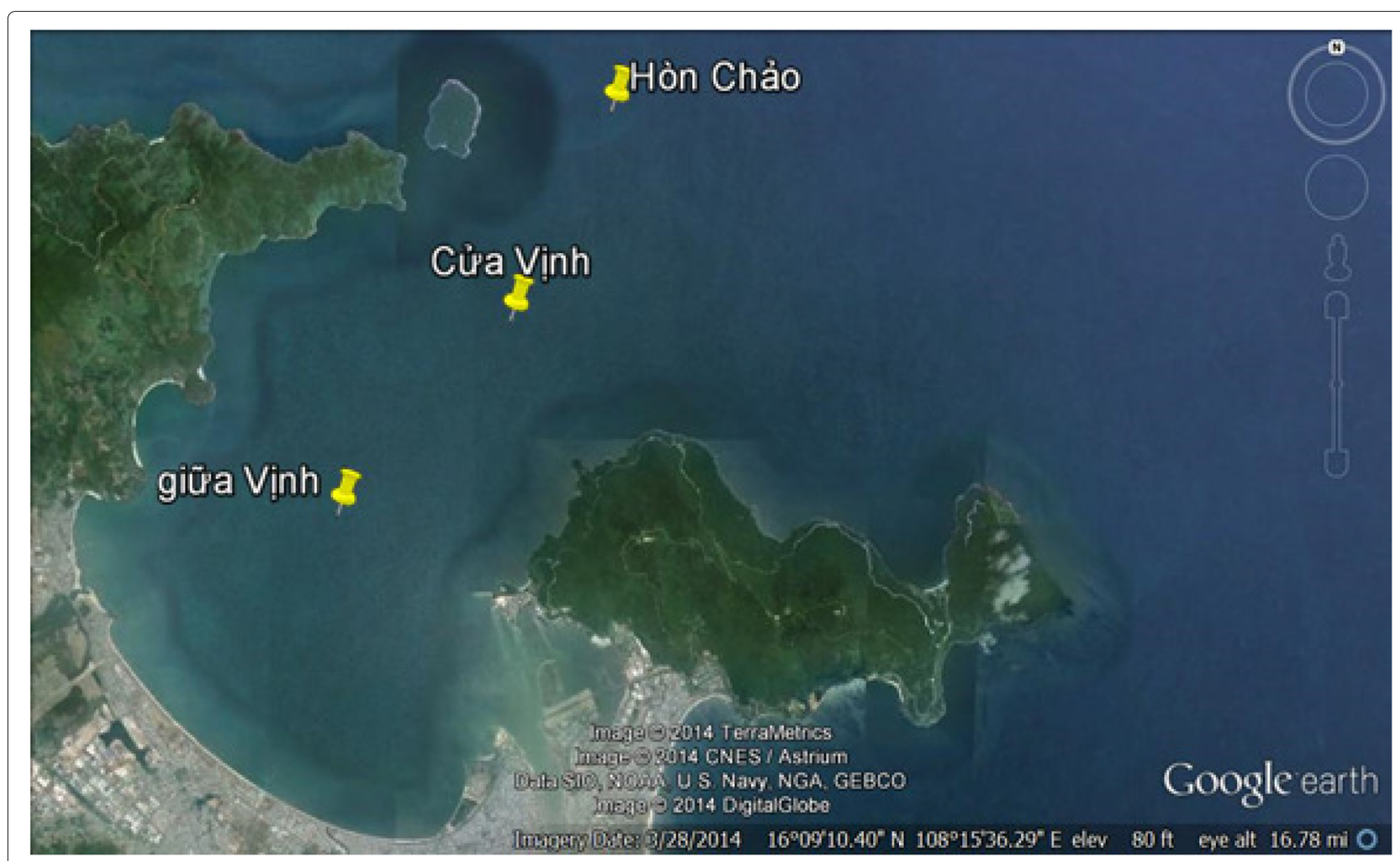

Fig. 4 Position of the measuring points in the simulated region at Danang Bay, Vietnam, reproduced from Google Earth (https://goo.gl/maps/ DJHVpRTejF82)

$$
\zeta=A_{0}+\sum_{i=1}^{n} A_{i} \cos \left(\omega_{i} t-g_{i}\right)
$$

where $A_{i}$ is the amplitude of the $i-t h$ wave, is its angular frequency and $g_{i}$ its phase. The tidal parameters are interpolated from the global parameter table.
Figure 5 shows the simulation results for the tidal flow in Danang Bay at the rising phase (May 1st 2014, 18:00) and at the receding phase (May 2nd 2014, 03:00). In general, the tidal flow is very small at all tidal phases.

Figure 6 shows the simulation results of water level fluctuation as compared to the measured data analysis using

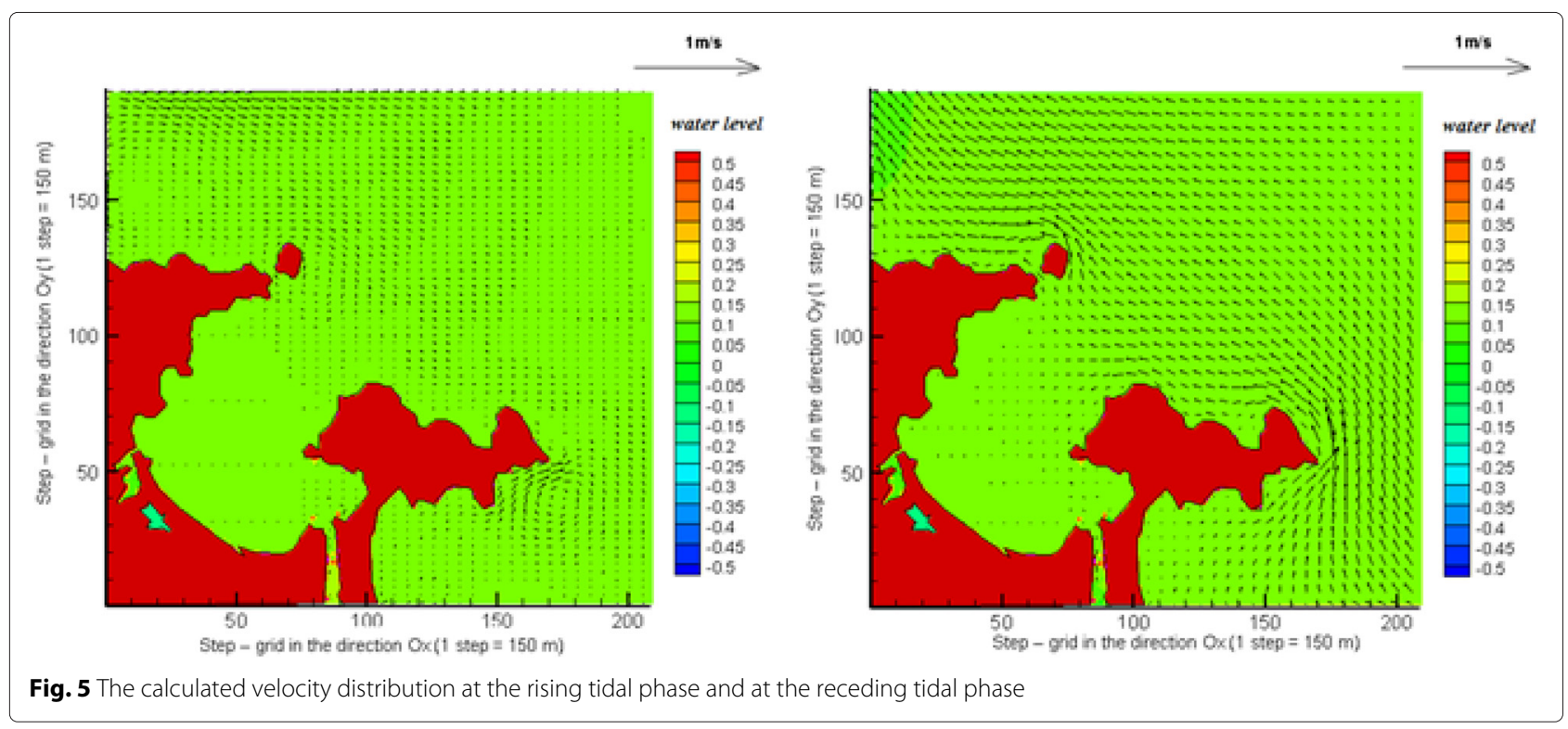



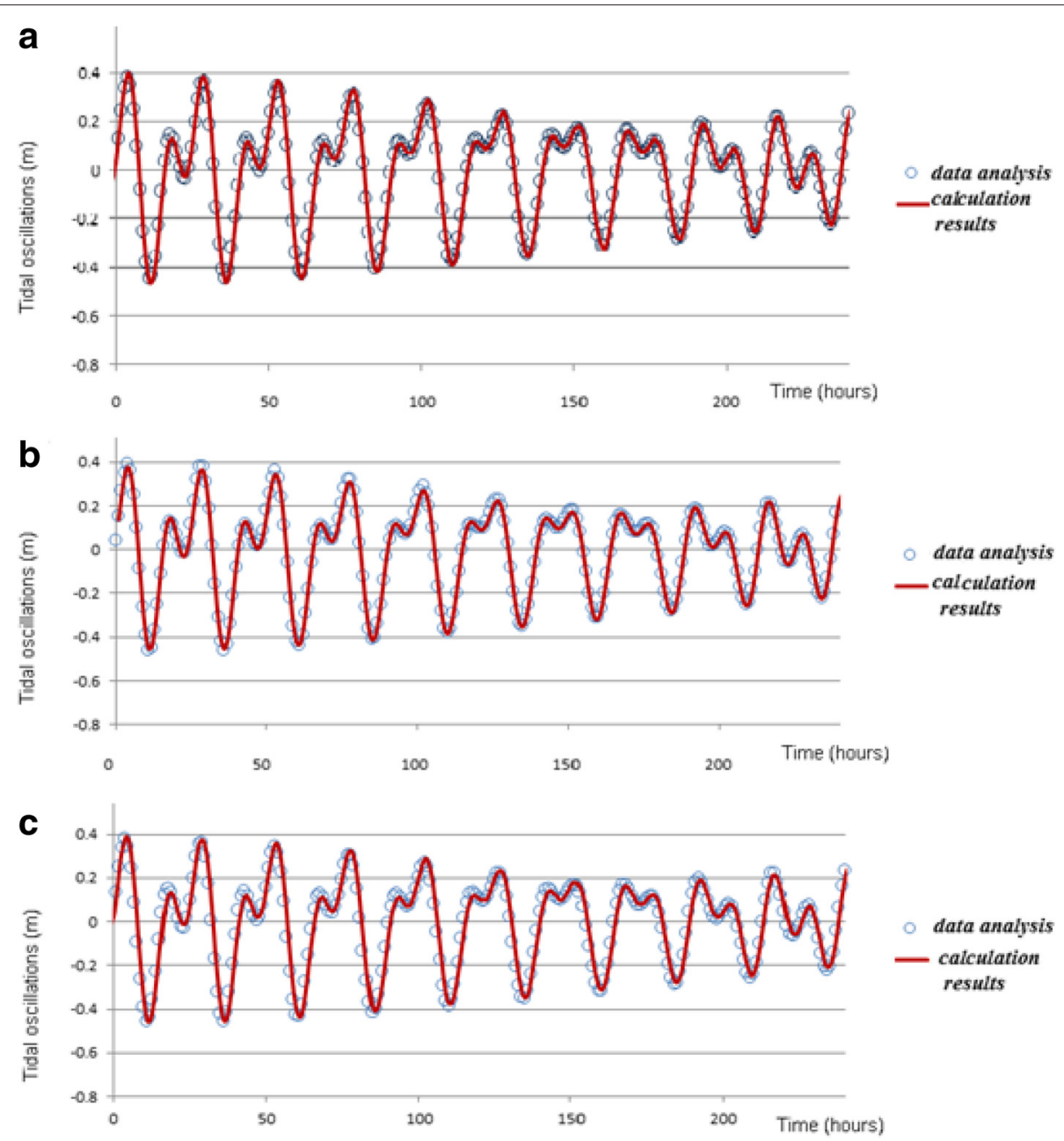

Fig. 6 Simulation results of water level fluctuation based on the non-hydrostatic shallow water model. The measured data are shown with circles. (a. Hon Chao, b. Cua Vinh, c. Giua Vinh)

the harmonic constants. The discrepancy is negligibly small.

\section{Conclusions}

This paper has presented a formulation of a new general shallow-water equations augmented by non-hydrostatic pressure effect having parameter $\alpha$ related to the depthintegrated non-hydrostatic pressure. The total pressure is decomposed into the hydrostatic and the non-hydrostatic components. The resulting equations are integrated in several steps. In the numerical model, the explicit method is applied to the hydrostatic component of the pressure, and the formulation of the Poisson equation for implicitly solving the non-hydrostatic pressure. This paper has demonstrated the versatility and robustness of the effect of non-hydrostatic pressure for simulating tidal flow. The simulation results were compared with the experimental data for wave propagation in a flume. The tradional hydrostatic-pressure model and our nonhydrostatic pressure model were both compared with eight experimental data sets. The results of our nonhydrostatic model exhibits agreement with the experimental data and is better than the traditional hydrostatic model. A numerical model was derived and successfully applied to simulate the tidal flow in Danang Bay, Vietnam. The simulation results for this last case were also compared with measurement data to show the applicability to the tidal problem.

In the model of Yamazaki et al. [19], the non-hydrostatic component of the pressure has been incororated into the average value of the pressure component at the bottom and at the surface. In our model, this component has been represented by introducing some function (10) and the parameter $\alpha$. This shew that the calculation results are better than the model of Yamazaki et al. and it is possible for application widely in other problems of the flow.

In the future, the effect of the rotation of the earth and the density variation of water with depth will tackled for simulating in propagation of the waves. The consideration of the effect of these components is necessary and is 
expected to improve the accurately in approximation. We also propose further development of other possible conditions applied to simulation in other problems, such as tides, storm surges and tsunamis, etc.

\section{Acknowledgements}

This study was supported by Ministry of Natural Resources and Environment of the Socialist Republic of Vietnam under Grant TNMT.2016.05.11. We are grateful to Hanoi University of Natural Resources and Environment for supporting us during the implementation of this study.

\section{Author details}

${ }^{1}$ Hanoi University of Natural Resources and Environment, Hanoi, Vietnam.

${ }^{2}$ Vietnam Institute of Seas and Islands, Trung Kinh, Cau Giay, Hanoi, Vietnam.

${ }^{3}$ Hanoi University of Science, Vietnam National University, Hanoi, Vietnam.

Received: 11 February 2015 Revised: 7 November 2015

Accepted: 28 December 2015

Published online: 10 March 2016

\section{References}

1. Briggs, MJ, Synolakis, CE, Harkins, GS, Green, DR: Laboratory experiments of tsunami runup on a circular island. Pure Appl. Geophys. 144, 569-593 (1995)

2. Casulli, V: A semi-implicit finite difference method for non-hydrostatic free surface flows. Int. J. Numer. Methods Fluids. 30(4), 425-440 (1999)

3. Chen, Q, Kirby, JT, Dalrymple, RA, Kennedy, AB, Chawla, A: Boussinesq modeling of wave transformation, breaking, and runup. II: 2D. J. Waterw. Port Coast. Ocean Eng. 126(1), 48-56 (2000)

4. Dodd, N: Numerical model of wave run-up, overtopping, and regeneration. J. Waterw. Port Coast. Ocean Eng. 124(2), 73-81 (1998)

5. Horrillo, J, Kowalik, Z, Shigihara, Y: Wave Dispersion Study in the Indian Ocean-Tsunami of December 26, 2004. Marine Geodesy. 29(1), 149-166 (2006)

6. Imamura, F: Review of tsunami simulation with a finite difference method. In: Yeh, H, Liu, P, Synolakis C (eds.) Long-wave Runup Models, pp. 25-42. World Scientific, Singapore, (1996)

7. Keller, HB: A new difference scheme for parabolic problems. In: Hubbard, $B$ (ed.) Numerical Solutions of Partial Differential Equations, II, pp. 327-350. Academic Press, New York, (1971)

8. Kowalik, Z, Knight, W, Logan, T, Whitmore, P: Numerical modeling of the global tsunami: Indonesian tsunami of 26 December 2004. Sci. Tsunami Hazards. 23(1), 40-56 (2005)

9. Kowalik, Z, Murty, TS: Numerical Modeling of Ocean Dynamics. World Scientific, Singapore (1993)

10. Lynett, PJ, Wu, TR, Liu, PLF: Modeling wave runup with depth-integrated equations. Coast. Eng. 46(2), 89-107 (2002)

11. Mader, CL: Numerical Modeling of Water Waves. CRC Press, New York (1993)

12. Madsen, PA, Srensen, OR, Schaffer, HA: Surf zone dynamics simulated by a Boussinesq type model. Part I. Model description and cross-shore motion of regular waves. Coast. Eng. 32(4), 255-287 (1997)

13. Peregrine, DH: Long waves on a beach. J. Fluid Mech. $\mathbf{2 7}(4), 815-827$ (1967)

14. Stelling, GS, Duinmeijer, SPA: A staggered conservative scheme for every Froude number in rapidly varied shallow water flows. Int. J. Numer. Methods Fluids. 43(12), 1329-1354 (2003)

15. Stelling, GS, Zijlema, M: An accurate and efficient finite-difference algorithm for non-hydrostatic free-surface flow with application to wave propagation. Int. J. Numer. Methods Fluids. 43(1), 1-23 (2003)

16. Synolakis, CE: The runup of solitary waves. J. Fluid Mech. 185, 523-545 (1987)

17. Van der, Vorst, HA: Bi-CGSTAB: a fast and smoothly converging variant of $\mathrm{Bi}-\mathrm{CG}$ for the solution of nonsymmetric linear systems. SIAM J. Sci. Stat. Comput. 13(2), 631-644 (1992)

18. Walters, RA: A semi-implicit finite element model for non-hydrostatic (dispersive) surface waves. Int. J. Numer. Methods Fluids. 49(7), 721-737 (2005)
19. Yamazaki, Y, Kowalik, Z, Cheung, KF: Depth-integrated, non-hydrostatic model for wave breaking and run-up. Int. J. Numer. Methods Fluids. 61(5), 473-497 (2008)

20. Yamazaki, Y, Wei, Y, Cheung, KF, Curtis, GD: Forecast of tsunamis generated at the JapanKurilKamchatka source region. Nat. Hazards. 38(3), 411-435 (2006)

21. Zangping, Wei, Yafei, Jia: A depth-integrated non-hydrostatic finite element model for wave propagation. Int. J. Numer. Model. Fluids. 73, 976-1000 (2013)

22. Zhou, JG, Causon, DM, Mingham, CG, Ingram, DM: The surface gradient method for the treatment of source terms in the shallow-water equations. J. Comput. Phys. 168(1), 1-25 (2001)

23. Zijlema, M, Stelling, GS: Efficient computation of surf zone waves using the nonlinear shallow water equations with non-hydrostatic pressure. Coast. Eng. 55(10), 780-790 (2008)

\section{Submit your manuscript to a SpringerOpen ${ }^{\circ}$ journal and benefit from:}

- Convenient online submission

- Rigorous peer review

- Immediate publication on acceptance

- Open access: articles freely available online

- High visibility within the field

- Retaining the copyright to your article

Submit your next manuscript at $>$ springeropen.com 